\title{
Group rearing leads to long-term changes in locomotor activity of Drosophila males
}

\author{
Anna A. Panova, Julia V. Bragina, Larisa V. Danilenkova, Natalia G. Besedina, \\ Elena A. Kamysheva, Sergey A. Fedotov, Nikolay G. Kamyshev
}

Pavlov Institute of Physiology, Russian Academy of Sciences, St. Petersburg, Russia; nkamster@gmail.com

Received 17 August 2013; revised 21 September 2013; accepted 6 October 2013

Copyright (C) 2013 Anna A. Panova et al. This is an open access article distributed under the Creative Commons Attribution License, which permits unrestricted use, distribution, and reproduction in any medium, provided the original work is properly cited.

\begin{abstract}
Previous social experience may affect subsequent behavior. It was shown by other authors that Drosophila melanogaster males kept individually are more aggressive and sexually active than males kept in a group. In the present study, we tested the locomotor activity of individual males and females previously reared either individually, or in a group. We found that keeping 20 young males for three days together led to a strong long-term (up to 5 days) reduction in their further locomotor activity as individuals. Rearing of young males in groups of other sizes $(2,5$, 10 , and 30 ) produced a smaller or no after-effect. At the same time, we have not found any difference in subsequent behavior of individual females previously kept either individually, or in a group. We suppose that in a group, flies learned to suppress their locomotor activity to prevent unpleasant contacts with other animals (operant learning). It seems that in males this learning is more efficient because of the higher level of aggression producing the stronger negative reinforcement.
\end{abstract}

Keywords: Drosophila; Social Experience; Group Situation; Locomotor Activity

\section{INTRODUCTION}

It is known that previous social experience can modify further behavior. It is true not only for the social animals but for animals without developed sociality, like Drosophila, too. Today little is known about social interactions and their consequences in subsequent Drosophila behavior. In addition to better understanding of animal behaveior, such studies may have implications for creation of animal models of human psychiatric disorders, where social isolation or interaction is a contributing factor.

The main interactions between Drosophila individuals are presented by courtship, aggressive and aggregation behaviors. Flies cooperate into aggregation being attracted by volatile pheromone cis-vaccenyl acetate [1-2]. It was shown in Drosophila mutants that keeping flies in a group leads to significant life extension [3]. But it is not still clear what is more stressful for Drosophila: staying alone or in a group.

It was shown that each fly prefers to maintain individual distance free from other flies [4]. Close contacts are accompanied by kicks, wing threats and jumps [5-6]. Also, in a group situation flies spent more time in preening, this behavior was considered as a visual signal preventing contacts [7]. Previously, we found that in a group situation, Drosophila melanogaster females showed two unconditioned responses: 1) running from the fly breaking the individual distance, that increased their run frequency, and 2) stopping when continuation of the run threatened collision with another female, that decreased their run duration. By the trial and error method (operant learning) females learn to decrease their locomotor activity that results in reduction of unpleasant contacts with other individuals [8]. After isolation from a group situation, females restored their level of activity very rapidly showing fast extinction of operant conditioning. However, the classical conditioning in heterogeneous environment was found, when threat from other flies it was associated with context stimuli, and further presentation of context in absence of other flies was sufficient to inhibit activity [8].

One more attempt to understand the process of cooperation of flies in a group was made by Tinette group. [9]. They suggest that flies may act in a cooperative manner to perform food searching, namely that there are two types of flies: "primers" and "followers". The former investigate food substrate and choose the best one. The latter, "followers", move to that food source and aggre- 
gate there. In other studies it was shown that keeping males in a homogenous group leads to a long-term depression of their aggressive and sexual behaviors [1014,6]. Females, if they have a choice between a male previously kept in a group and a male kept individually, choose the more active one, i.e. kept individually. Also it was found that isolation of males from conspecifics led to increase of homosexual behavior. The probable reason is the modification of cuticle pheromone composition. In two studies it was shown that chemical signaling is affected, besides light-dark cycle and genotype, by the social environment [15-17].

The problem of influence of social context and social experience on behavior is widely studied in laboratory animals [18-22].

A number of investigations have shown the importance of social environment for rodents. It was revealed that rearing in social isolation during pubertal period could lead to hyperactivity, increased aggressive behaveior, reduced habituation, novel object recognition and resulted in reduction of medial prefrontal cortex volume, cytoskeletal alteration in hippocampus and changes in CREB expression and dopamine and serotonin turnover in different parts of brain [23].

\section{MATERIALS AND METHODS}

\subsection{Flies}

Drosophila melanogaster wild-type line Canton-S was used. Males and females were collected during 3 hours after eclosion by aspiration. All flies were kept in $25 \times$ $95 \mathrm{~mm}$ vials containing standard yeast-semolina-raisinsugar medium under 12:12 light-dark cycle at $25^{\circ} \mathrm{C}$ and relative humidity of $60 \% \pm 5 \%$. Collected males and females were kept either individually (control), or in groups (experiment) for 3 days. Then experimental males were separated from each other. To examine the influence of group size $(2,5,10,20$, or 30 individuals per vial) on subsequent locomotor activity of experimental males they were tested individually immediately after separation. Experimental males used in investigation of the after-effect duration were kept in groups of 20 . The control and experimental males were tested daily for 8 days (independent samples for each day). Experimental females after keeping in groups of 20 were tested individually immediately after isolation.

\subsection{Locomotion Analysis}

All experiments were made from 11 a.m. to 5 p.m. Males were introduced individually by aspiration into chambers (15 $\mathrm{mm}$ diameter, $5 \mathrm{~mm}$ height) made in a plexiglass plates. The plates were positioned under two web cameras, watching behavior of 20 flies simultaneously, using the original program "Drosophila tracks" (C)
N. G. Kamyshev) allowing to monitor and to analyze the time course of various parameters of locomotion. The main parameter is activity index, i.e. the percent of time spent in locomotion. In case of examination of group size effect in males and experiments with females, registration lasted for 1 hour with statistical analysis of 12 successive 5-min periods. When duration of the after-effect of group keeping was examined, daily registration time was 5 hours with statistical analysis of 10 successive 30-min periods (wet filer paper was placed into experimental chambers in this case). Also we computed the joint activity index for last 5 periods (2.5 hours) of 5-hours testing period.

\section{RESULTS AND DISCUSSION}

\subsection{Dependence of Subsequent Locomotor Activity in Individuals, Previously Kept in a Group, on Group Size}

First of all, we checked the influence of group size on further locomotor activity of males separated from a group to choose the one with maximum after-effect (Figure 1). We kept Drosophila males alone or in a group of 2, 5, 10, 20 or 30 flies for 3 days after eclosion. Then we tested locomotor activity in individuals separated from a group. The largest effect of previous social experience was found for flies kept in groups of 20 individuals.

Reduction of locomotor activity in line $1 \rightarrow 5 \rightarrow 10 \rightarrow 20$ flies is quite good predictable. The more flies, the more frequent collisions between them. That, in turn, increases the magnitude of changes in their behavior.

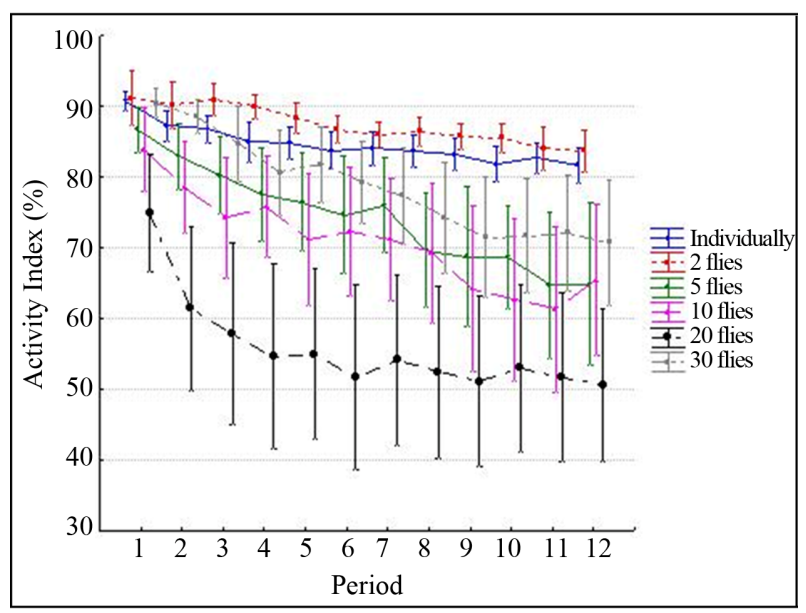

Figure 1. Influence of group size on further locomotor activity of males separated from a group. Effect of group keeping for 3 days depends on group size. 20-flies group showed the largest difference from the control (flies kept individually). Y axis: activity index (percent of time spent in locomotion). $\mathrm{X}$ axis: 5 -min periods of observation. Vertical bars show 95\% confidence intervals. $\mathrm{N}=28-31$ for each point. 
One exclusion from this rule is no or even opposite after-effect of group keeping, when a group consists only of two males. When two males meet in a vial, it can be assumed, they prefer to stay separately. Probably, every accidental meeting stimulates them to run away. As flies do not encounter many obstacles, they need not stop running. As a result we can see a slight activation of activity in comparison to males kept individually. In contrast, the group density of 5 (in given experimental conditions) is already sufficient to provide inhibition of locomotor activity in a group situation.

The most surprising are the meanings of locomotor activity of flies kept in 30-individuals group. Although at the beginning of observation these flies show no difference from control, by the end of 1 hour their locomotor activity became less than activity of individual ones. Nevertheless, they are more active than flies kept in 20-flies group.

If to agree (see Introduction and Conclusion) that inhibition of locomotor activity after group rearing results from learning, this may imply that group density of 30 flies per vial leads to cognitive disfunctions. The reasons for that may consist in the worsening of life conditions due to great number of negative interactions including loss in concurrency for food resources.

\subsection{Previous Group Keeping Leads to Changes of Male, but not Female Locomotor Activity}

To verify the previous data about the lack of after-effect of group keeping in females [8], we compared locomotor activity in females kept for 3 days after eclosion either individually, or in a 20-flies group (Figure 2). Our results confirm the fact that females do not change their behavior after group keeping. The effects of previous social experience, is yet poorly investigated. In rodents, it was shown that rearing in social isolation during pubertal period led to hyperactivity, reduced habituation, novel object recognition and floating time in forced swim tests [23]. But all these effects concern both males and females. Our results show that consequences of social experience in Drosophila are quite different for two sexes. It may be assumed that Drosophila females are less aggressive than males and provoke collisions with other flies less frequently (normally, they do not show homosexual behavior).

\subsection{Keeping Drosophila Males in a Group Leads to a Long-Term Locomotion Inhibition}

To investigate the duration of behavioral changes after group rearing we kept males in 20-flies groups during 3 days. Then we isolated them from each other and tested experimental and control flies immediately and for next 7 days, each day for 5 hours. Because the most signifycant difference between experimental and control flies were observed during last 2.5 hours, the joint activity index for this time interval was analyzed (Figure 3).

There is a great difference between males kept individually and males kept in a group, which retains until 5 days after isolation of experimental males. Importantly, these behavioral changes are temporary. This excludes the possibility that they are based on alteration of brain structures that was found in rodents [24,25]. According to our previous investigations [8] we suppose that this locomotor suppression is the result of operant learning

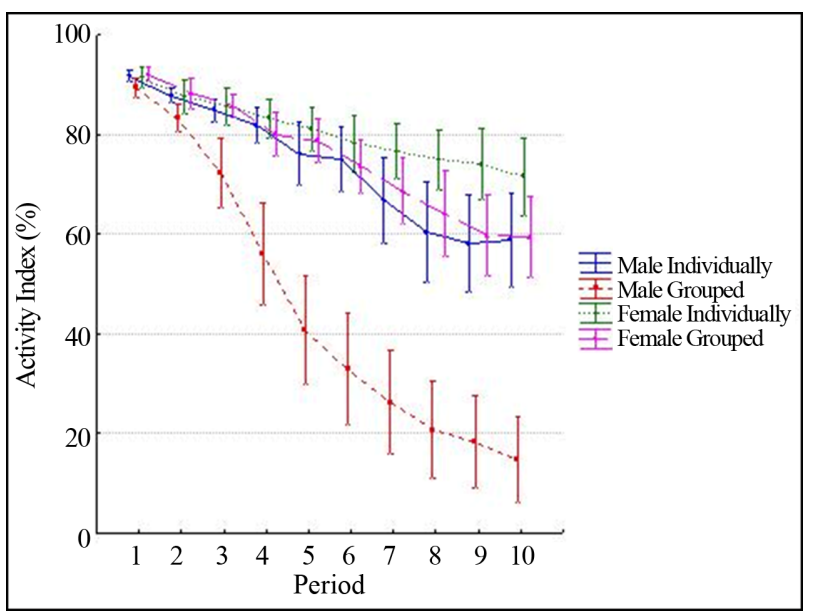

Figure 2. Absence of after-effect of group keeping in Drosophila females. In contrast to Drosophila males, females do not change their locomotor activity after 3-days keeping in a group. $\mathrm{Y}$ axis: activity index (percent of time spent in locomotion). $\mathrm{X}$ axis: 5-min periods of observation. Vertical bars show 95\% confidence intervals. $\mathrm{N}=40-50$ for each point.



Figure 3. Group keeping leads to long-term changes in Drosophila male locomotor activity. Y axis: activity index calculated for the last 2.5 hours of 5-h observation. $\mathrm{X}$ axis: days after isolation from a group. Vertical bars show $95 \%$ confidence intervals. $\mathrm{N}=40-50$ for each point. 
occurred in a group. Being in a group situation, flies learn to avoid contacts with other individuals because they are accompanied with threats and fighting playing a role of negative reinforcement. As a result, flies prefer to stay in rest than being active.

\section{CONCLUSIONS}

Previous studies have shown that keeping Drosophila males in a group leads to increase of aggression and decrease of sexual activity in comparison with males kept individually $[10-14,6]$. In our research we showed that previous social experience is extremely important for further behaviour. Keeping males together for 3 days in groups of 20 flies leads to a long-term strong reduction of their locomotor activity maintained for 5 days after separation. This may be a result of operant learning similar to learning described earlier [Kamyshev et al. 2002] in females. It was shown that in a group the flies try to avoid close unpleasant contacts accompanied by kicks and wing threats from other insects. Therefore, they run from the individuals, which came too near, and stop their running to prevent meeting with another female. Trial and error learning forces them to suppress their locomotor activity and stay at rest; as a result they have a less number of unpleasant contacts. But in the case of females this learning leads to no after-effects in absence of the appropriate contextual cues. Males, in contrast, possibly due to more intensive aggression, retain the altered behavior for a long time independently of the context, in which they experienced aggression from other individuals.

Ascertainment of underlying physiological and molecular mechanisms of these phenomena may illuminate the basic processes of Drosophila social interactions. These results will help to standardize laboratory protocols of Drosophila housing. Also, the fruit fly, as well as rodents, can be used as a model for investigation of social stress and its effects on development of neuropsychiatric disorders.

\section{ACKNOWLEDGEMENTS}

This study was supported by grants No. 13-04-02153 and No. 13-0412030 from Russian Foundation for Basic Researches, by Programs of Presidium of the Russian Academy of Sciences No. 7 and No. 30.

\section{REFERENCES}

[1] Threlkeld, S.F., Procwat, R.A., Abbot, K.S. and Yeung, A.D. (1974) Genetically based behavior patterns in Drosophila. Nature, 247, 232-233.

http://dx.doi.org/10.1038/247232a0

[2] Bartelt, R., Schaner, A. and Jackson, L. (1985) Cis-vaccenyl acetate as an aggregation pheromone in Drosophila melanogaster. Journal of Chemical Ecology, 11, 1747-1756. http://dx.doi.org/10.1007/BF01012124

[3] Ruan, H. and Wu, C. F. (2008) Social Social interactionmediated lifespan extension of Drosophila $\mathrm{Cu} / \mathrm{Zn}$ superoxide dismutase mutants. Proceedings of the National Academy of Sciences of the United States of America, 21, 7506-7510. http://dx.doi.org/10.1073/pnas.0711127105

[4] Sexton, O.J. and Stalker, H.D. (1961) Spacing patterns of female D. paramelanica. Animal Behaviour, 9, 77-81. http://dx.doi.org/10.1016/0003-3472(61)90053-7

[5] Manning, A. (1959) The sexual behaviour of two sibling Drosophila. Behaviour, 15, 123-145. http://dx.doi.org/10.1163/156853960X00133

[6] Bastock, M. and Manning, A. (1955) The courtship of Drosophila melanogaster. Behaviour, 8, 85-111. http://dx.doi.org/10.1163/156853955X00184

[7] Connolly, K.J. (1968) The social facilitation of preening behaviour in D. melanogaster. Animal Behaviour, 16, 385-391. http://dx.doi.org/10.1016/0003-3472(68)90023-7

[8] Kamyshev, N.G., Smirnova, G.P., Kamysheva, E.A., Nikiforov, O.N., Parafenyuk, I.V. and Ponomarenko, V.V. (2002) Plasticity of social behavior in Drosophila. Neuroscience and Behavioral Physiology, 32, 401-408. http://dx.doi.org/10.1023/A:1015832328023

[9] Tinette, S., Zhang, L. and Robichon, A. (2004) Cooperation between Drosophila flies in searching behavior. Genes, Brain and Behavior, 3, 39-50. http://dx.doi.org/10.1046/j.1601-183x.2003.0046.x

[10] Hoffmann, A.A. (1990) The influence of age and experience with conspecifics on territorial behavior in Drosophila melanogaster. Journal of Insect Behavior, 3, 1-12. http://dx.doi.org/10.1007/BF01049191

[11] Svetec, N. and Ferveur, J.-F. (2005) Social experience and pheromonal perception can change male-male interactions in Drosophila melanogaster. The Journal of Experimental Biology, 208, 891-898. http://dx.doi.org/10.1242/jeb.01454

[12] Maynard, S.J. (1956) Fertilly, mating behavior and sexual selection in Drosophila subobscura. Journal of Genetics, 54, 261-279. http://dx.doi.org/10.1007/BF02982781

[13] Kim, Y.-K., Ehrman, L. and Koepfer, H.R. (1996) Developmental isolation and subsequent adult behavior of Drosophila paulistorum. I Survey of the six semispecies. Behavior Genetics, 22, 545-556. http://dx.doi.org/10.1007/BF01074307

[14] Kim, Y-K., Ehrman, L. and Koepfer, H.R. (1996) Developmental isolation and subsequent adult behavior of Drosophila paulistorum. II. Prior experience. Behavior Genetics, 26, 15-25. http://dx.doi.org/10.1007/BF02361155

[15] Kent, C., Azanchi, R., Smith, B., Formosa, A. and Levine, J. D. (2008) Social context influences chemical communication in D. melanogaster males. Current Biology, 18, 1384-1389. http://dx.doi.org/10.1016/j.cub.2008.07.088

[16] Krupp, J. J., Kent, C., Billeter, J.C., Azanchi, R., So, A. K., Schonfeld, J.A., Smith, B.P., Lucas, C. and Levine, J.D. (2008) Social experience modifies pheromone ex- 
pression and mating behavior in male Drosophila melanogaster. Current Biology, 18, 1373-1383. http://dx.doi.org/10.1016/j.cub.2008.07.089 doi:10.1016/j.cub.2008.07.089

[17] Ritchie, M.G. (2008) Behavioural genetics: The social fly. Current Biology, 23, 862-864.

http://dx.doi.org/10.1016/j.cub.2008.08.009

[18] Galef, B.G. Jr. and Giraldeau, L. A. (2001) Social influences on foraging in vertebrates: Causal mechanisms and adaptive functions. Animal Behaviour, 61, 3-15. http://dx.doi.org/10.1006/anbe.2000.1557

[19] Free, J.B. (1987) A social insect: the biology of the honey bee. Science, 238, 1591-1592. http://dx.doi.org/10.1126/science.238.4833.1591

[20] Rosengaus, R.B., Jordan, C., Lefebvre, M.L. and Traniello, J.F. (1999) Pathogen alarm behavior in a termite: A new form of communication in social insects. Naturwissenschaften, 86, 544-548. http://dx.doi.org/10.1007/s001140050672

[21] Rull, J, Procopy, R.J. and Vargas, R.I. (2003) Effects of conspecific presence on arrival and use of hosts in Ceratitis capitata flies. Journal of Insect Behavior, 16, 329-346.
http://dx.doi.org/10.1023/A:1024871908372

[22] Coolen, I., Dangles, O. and Casas, J. (2005) Social learning in noncolonial insects? Current Biology, 15, 19311935. http://dx.doi.org/10.1016/j.cub.2005.09.015

[23] Kercmar, J., Büdefeld, T., Grgurevic, N., Tobet, S.A. and Majdic, G. (2011) Adolescent social isolation changes social recognition in adult mice. Behavioural Brain Research, 216, 647-651. http://dx.doi.org/10.1016/j.bbr.2010.09.007

[24] Schubert, M. I., Porkess, M.V., Dashdorj, N., Fone, K.C. and Auer, D.P. (2009) Effects of social isolation rearing on the limbic brain: A combined behavioral and magnetic resonance imaging volumetry study in rats. Neuroscience, 159, 21-30. http://dx.doi.org/10.1016/j.neuroscience.2008.12.019

[25] Bianchi, M., Fone, K.F., Azmi, N., Heidbreder, C.A., Hagan, J.J. and Marsden, C.A. (2006) Isolation rearing induces recognition memory deficits accompanied by cytoskeletal alterations in rat hippocampus. European Journal of Neuroscience, 24, 2894-2902. http://dx.doi.org/10.1111/j.1460-9568.2006.05170.x 